\title{
DNA Sequencing with TEM
}

\author{
David C. Bell ${ }^{1}$, W. Kelley Thomas ${ }^{2}$, Katelyn Murtagh ${ }^{3}$, and William R. Glover ${ }^{3}$ \\ 1. School of Engineering and Applied Sciences, and Center for Nanoscale Systems, \\ Harvard University, Cambridge, Massachusetts 02138 \\ 2. Hubbard Center for Genome Studies, University of New Hampshire, Durham NH 03824 \\ 3. ZS Genetics, North Reading MA, 01864
}

We are on the cusp of a new age in medicine. Genetic researchers have provided the hope of resolving some of the worst diseases plaguing mankind. However, this promise is waiting for faster, cheaper and more accurate means of genetic analysis. Recently there have been evolutions and revolutions in DNA sequencing technologies. Some sequencing technologies use gene chips such as the DNA microarray used in molecular biology and in medicine. It consists of an arrayed series of thousands of microscopic spots of DNA oligonucleotides, called features. Since an array can contain tens of thousands of probes, a microarray experiment can accomplish many genetic tests in parallel. Therefore, arrays have dramatically accelerated many types of investigations. Another possible method is nanopore sequencing which has to do with what occurs when the nanopore is immersed in a conducting fluid and a potential (voltage) is applied across it [1]: under these conditions a slight electric current due to conduction of ions through the nanopore can be observed. If single bases or strands of DNA pass (or part of the DNA molecule passes) through the nanopore, the process can create a change in the magnitude of the current through the nanopore.

However one significant draw back of DNA microarrays and nanopore sequencing is that the "read" length is limited by the application of the underlying technology. The revolutionary approach described in this paper is based on "direct reading" includes using an imaging technology platform and preparation of suitable labeled DNA samples (Figure 1) by stretching out the double helix that then offers the ability to take direct images of genetic material identifying its labeling with one or a few key unique identifying labels. This platform has the potential to be used in a number of different applications in genetic analysis. The development of these systems has the promise to provide high throughput and low operating costs. The idea is simple: to sequence DNA by taking digital images of the labeled DNA bases with a high-resolution electron microscope (Figure 2), with the eventual aim being a fully automated imaging system. In this way a sequence thousands of base-pairs long can be read directly from the image with developed pattern recognition software.

For comparison we present results imaged with a variety of (S)TEM systems including a monochromated aberration-corrected TEM and STEM as well as a low cost TEM system. The completed system is expected to read a complete haploid of human genome in days, perhaps hours, with $4 \mathrm{X}$ coverage, at a cost in the tens of thousands of dollars. The path to the $\$ 1,000$ or less genome will then be in sight.

\section{References}

1. M.J. Kim, M.W. Wanunu, D.C. Bell, A. Meller "Rapid Fabrication of Uniformly Sized Nanopores and Nanopore Arrays for Parallel DNA Analysis", Adv. Mat. 18 (23) (2006), pp. 31493153 

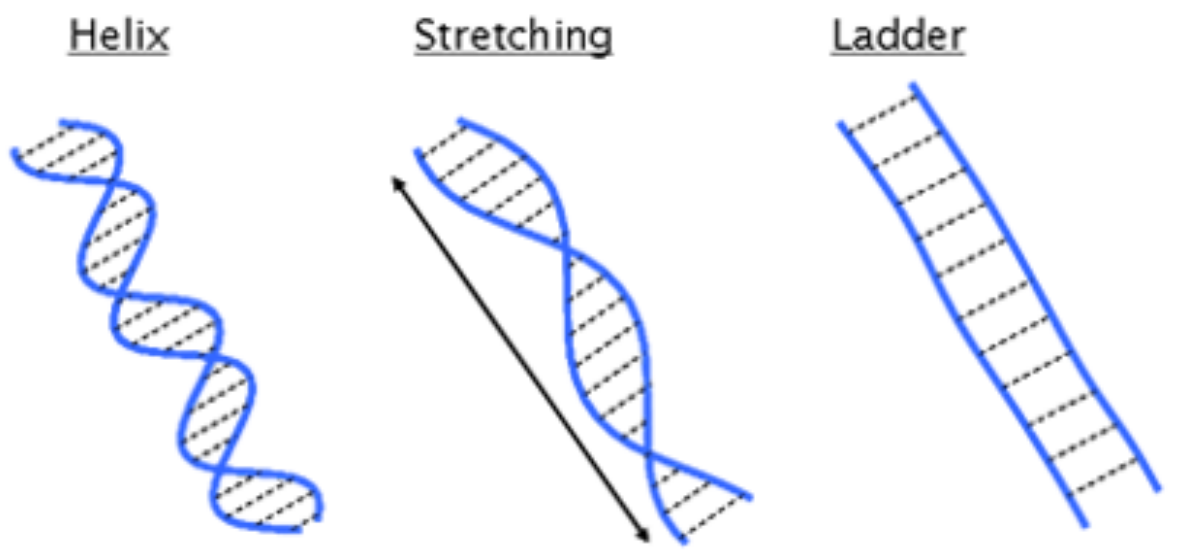

Figure 1. The fundamental sample preparation problem, stretching the DNA helix to lay flat, and allow comprehensive imaging; suitable labeling that is applied can then be used as a mechanism to "read" sequences.

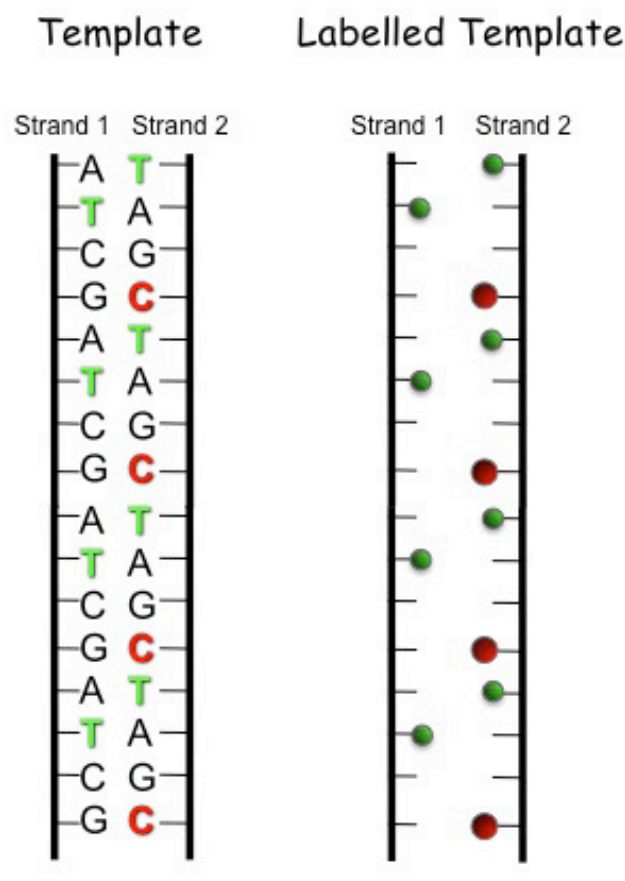
EM Image Overlaid with Labelled Template

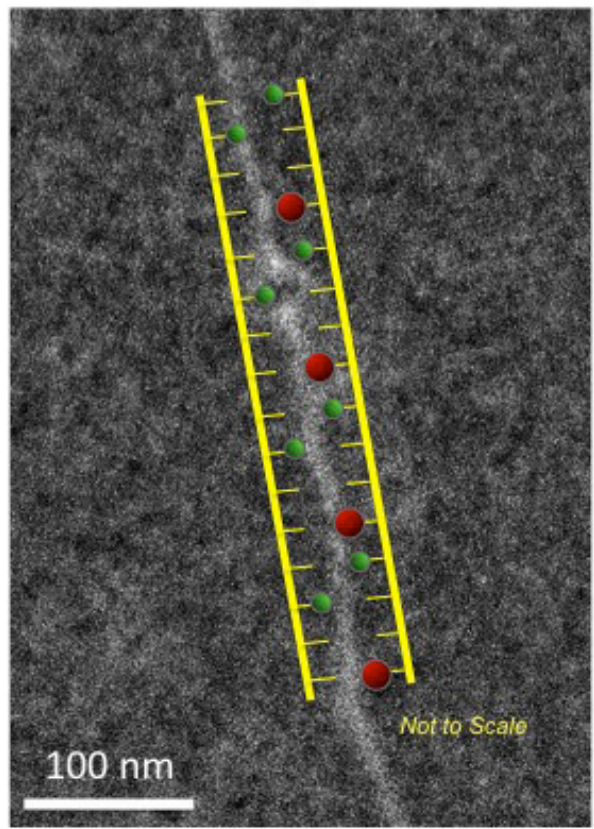

Figure 2. The labeled DNA template now able to be read directly using high-resolution electron microscopy, in this case an ADF STEM image is shown. 